\title{
Alleviation of Cd-induced growth improvement by exogenous melatonin pre-treatment in Abutilon theophrasti Medicus
}

\author{
Tianpeng $\mathrm{GAO}^{1,5^{*}}$, Jingwen $\mathrm{FU}^{2}$, Mingbo ZUO ${ }^{2}$, Haoming Wang ${ }^{3}$, Xiao-Xiao Liu ${ }^{1}$, Hai-Juan Li ${ }^{1}$, Yuan-Yuan Shen ${ }^{1}$, Yingli \\ $\mathrm{YANG}^{2}$, Dang-hui $\mathrm{XU}^{4}$, and Yueqing $\mathrm{He}^{3}$ \\ ${ }^{1}$ School of Biological and Environmental Engineering, Xi'an University, Xi'an710065, China \\ ${ }^{2}$ College of Geography and Environmental science, Northwest Normal University, Lanzhou 730070, China \\ ${ }^{3}$ School of Chemical and Biological Engineering, Lanzhou Jiaotong University, Lanzhou 730070, China \\ ${ }^{4}$ State Key Laboratory of Grassland Agro Ecosystems/School of Life Science, Lanzhou University, Lanzhou 730000, Gansu, China \\ ${ }^{5}$ College of Geography and Environmental Engineering, Engineering Center for Pollution Control and Ecological Restoration in Mining \\ of Gansu Province, Lanzhou City University, Lanzhou 730070, China
}

\begin{abstract}
The present work was aimed to study the effect of melatonin pre-treatments on oxidative stress modulation, cadmium (Cd) accumulation, and tolerance in Abutilon theophrasti Medicus under the hydroponic system. Application of substances that can modulate the harmful effects of $\mathrm{Cd}$ on plant yield and reduce its accumulation in the edible parts is of particular importance. Therefore, the Abutilon pre-treated with $0.1 \mathrm{mM}$ melatonin were exposed to $200 \mu \mathrm{M}, 400 \mu \mathrm{M}, 800 \mu \mathrm{M}$ Cd. Our results showed that $0.1 \mathrm{mM}$ melatonin led to positive effects on $200 \mu \mathrm{M}$ and $400 \mu \mathrm{M} \mathrm{Cd}$ tolerance, including a significant increase in root activity andperoxidase (POD) activity and a significant decrease in malondialdehyde (MDA) content. Withthe help of melatonin, the enrichment factor $(\mathrm{BCF})$ significantly decreased, reducing the content in the ground and roots. At least in our experiment conditions, this information appears to be useful for healthy food production.
\end{abstract}

\section{Introduction}

Among heavy metals, cadmium was a highly carcinogenic metal that could cause toxic reactions even at low concentrations $^{[1-2]}$. In plants, yellowing and growth shunt caused by $\mathrm{Cd}$ toxicity were easily to find symptoms. Higher toxicity would inhibit plant growth and lead to plant necrosis ${ }^{[3]}$. The toxicity of $\mathrm{Cd}$ affected plants by inhibiting carbon fixation and reducing chlorophyll content and photosynthetic activity ${ }^{[4]}$. Cd exposure in the soil induced osmotic stress in plants by minimizing the relative water content, stomatal conductance and transpiration of leaves, which led to plant physiological damages ${ }^{[5]}$. The toxicity of cadmium could lead to the excessive production of reactive oxygen species (ROS), and lead to damage to plant membranes and destruction of cell biomolecules and organelles ${ }^{[6]}$. Cadmium also reduced the absorption of iron and zinc by plants, leading to yellowing of leaves ${ }^{[7]}$. Generally, Cd interfered with the transportation and absorption of $\mathrm{Ca}, \mathrm{P}, \mathrm{Mg}, \mathrm{K}$, and $\mathrm{Mn}$.

Melatonin was a pleiotropic molecule, which had multiple functions in plants. It was mainly considered primarily an antioxidant with important actions in the control of reactive oxygen and nitrogen species, among other free radicals, and harmful oxidative molecules present in plant cells ${ }^{[8]}$. In addition, melatonin also participated in a variety of physiological functions, such as growth, rooting, seed germination, photosynthesis, and protection of plants from abiotic and biotic stresses. Due to the diversity of its effects, melatonin was also considered to be the master regulator of plants. Exogenous application of melatonin can improve the resistance of plants to abiotic stress, reduce cell damage, and increase the activity of antioxidant enzymes SOD, POD, CAT and APX ${ }^{[9-10]}$. Therefore, the protective effect of melatonin in abiotic stress might be an ideal target to improve the ability of plants to resist biotic stress. Researchers ${ }^{[11]}$ used melatonin to treat $\mathrm{Cd}$ stress in tomato (Solanum lycopersicum), and obtained melatonin-induced antioxidant activity, phytochelatin biosynthesis and $\mathrm{Cd}$ partitioning played a key role in enhancing the tolerance to $\mathrm{Cd}$ stress effect.

Here, we took Abutilon seedlings as the research object, and explored the effects of melatonin on its physiology and enrichment function under cadmium stress by adding $0.1 \mathrm{mmol} / \mathrm{L}$ melatonin to pre-treatment, in order to provided a scientific basis for phytoremediation of soil cadmium pollution, increased certain economic benefits while remediating cadmium contaminated soil, and ensured safe production.

* Corresponding author: zkgtp@163.com; ffjjww0224@163.com 


\section{Methods and materials}

\subsection{Design of experiment}

The seedling growth test was carried out in the greenhouse of Xi'an University. We put the sterilized Abutilon seeds in a hydroponic device filled with vermiculite, incubate for 7 days in a $25^{\circ} \mathrm{C}$ incubator. Then selected seedlings with the same height, transferring to the artificial simulated climate room with $1 / 2$ Hoagland nutrition solution. Maintain aeration during the cultivation process, the photoperiod was $12 \mathrm{~h} / 12 \mathrm{~h}$ (day/night), the temperature was $22^{\circ} \mathrm{C}$, the relative humidity was $80 \%$, and the light intensity was $30001 \mathrm{x}$, the nutrient solution was changed every 3 days. In order to eliminate the influence of cadmium ions in the water, ultrapure water was used to configure the nutrient solution throughout the hydroponic process, and the $\mathrm{pH}$ of the culture solution was adjusted to $5.8 \sim 6.0$ with $0.1 \mathrm{~mol} / \mathrm{L} \mathrm{HCl}$ or $1 \mathrm{~mol} / \mathrm{L} \mathrm{KOH}$.

After 15 days of seedling growth, add $0.1 \mathrm{mM}$ melatonin to the nutrient solution for treatment. After 24 hours of treatment, different concentrations of $\mathrm{CdCl}_{2}$ were added to the 5L nutrient at 8:00the next morning for stress. The experiment set up 8 treatments: control group T0 $(0$ MEL, 0 Cd), Cd stress group CK200, CK400, CK800 (0 MEL, 200Cd; 0 MEL, 400Cd; 0 MEL, 800Cd), control plus melatonin group T0 (0.1 MEL, $0 \mathrm{Cd})$ and melatonin plus $\mathrm{Cd}^{2+}$ stress groups T200, T400, T800 (0.1 MEL, 200Cd; 0.1 MEL, 400Cd; 0.1 MEL, 800Cd). Plant samples were collected for analysis and testing at 5 days of stress.

\subsection{Indicators and test methods}

The content of malondialdehyde (MDA), soluble protein (SP), chlorophyll (Chl) and peroxidase (POD) were determined according to the TTC method for measuring root activity ${ }^{[12]}$ and Chen method ${ }^{[13]}$.

Referring to the method of Wei ${ }^{[14]}$, the root system and the upper part of the root of the abutilon seedling were separated and dried at $105^{\circ}$ to constant weight. The dried tissue was ground and weighed. The $0.25 \mathrm{~g}$ sample was digested inadigester (Labtech Digiblock ED54, China) with $10 \mathrm{~mL} \mathrm{HNO}_{3}$. The obtained digestive liquid was refrigerated, transferred to a $50 \mathrm{~mL}$ volumetric flask, and diluted to the corresponding volume by adding $1 \% \mathrm{HNO}_{3}$. After dilution of the liquid by membrane filtration $(0.45 \mu \mathrm{m})$ to clean the pipe. The concentration of $\mathrm{Cd}$ was determined by ICP-MS (Agilent 7500X, USA). In acid digestion and $\mathrm{Cd}$ content determination, the standard product (GBW07603 GSV-2) was used as quality control product to analyze the content of $\mathrm{Cd}$ in different samples. The content of $\mathrm{Cd}$ in the standard was 0.38 $\mathrm{mg} \cdot \mathrm{kg}^{-1}$, and the analysis result was $0.376 \pm 0.011 \mathrm{mg} \cdot \mathrm{kg}^{-1}$.

Transfer Factor $(\mathrm{TF})=$ Heavy metal content in the above-ground part $(\mathrm{mg} / \mathrm{kg}) /$ heavy metal content in the underground part $(\mathrm{mg} / \mathrm{kg})$

Enrichment Factor (BCF) = Heavy metal concentration in plants $(\mathrm{mg} / \mathrm{kg}) /$ heavy metal concentration in Nutrient solution $(\mathrm{mg} / \mathrm{kg})$

\subsection{Data processing and analysis}

Statistical analyses and visualization were performed in SPSS 23.0, Origin 2018 for Windows. The data were mean \pm standard error of three repeats. LSD test was selected $(\mathrm{P}<0.05)$ for significance analysis of differences.

\section{Results and discussions}

\subsection{Effect of melatonin on activityof root under Cadmium Stress}

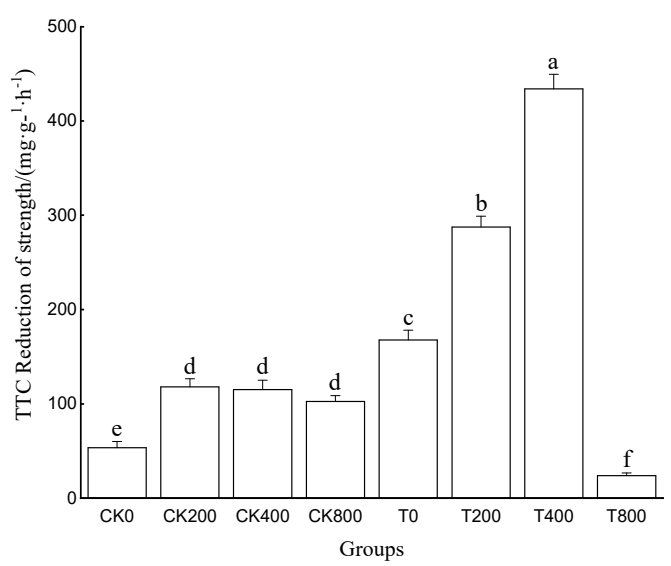

Figure 1. Effects of melatonin on the root activity of Abutilon theophrasti Medicus under $\mathrm{Cd}^{2+}$ stress

Note: Different lowercase letters indicate that there were significant differences between different stress concentrations $(\mathrm{P}<0.05)$, the same as below.

As shown in Figure 1, after 5 days of $\mathrm{Cd}$ stress treatment, root activity first increased and then decreased with increasing $\mathrm{Cd}$ stress treatment. Compared with CK400, the root activity of T400 increased by $276.58 \%$, and reached the maximum and the root activity of T800 decreased by $76.79 \%$. The results show that $0.1 \mathrm{mM}$ melatonin pre-treatment significantly $(\mathrm{P}<0.05)$ increased the root activity of seedlings under low concentration stress, which meant melatonin had an activating effect on the growth and physiological of abutilon roots.

\subsection{Effect of melatonin on content of SP under cadmium stress}

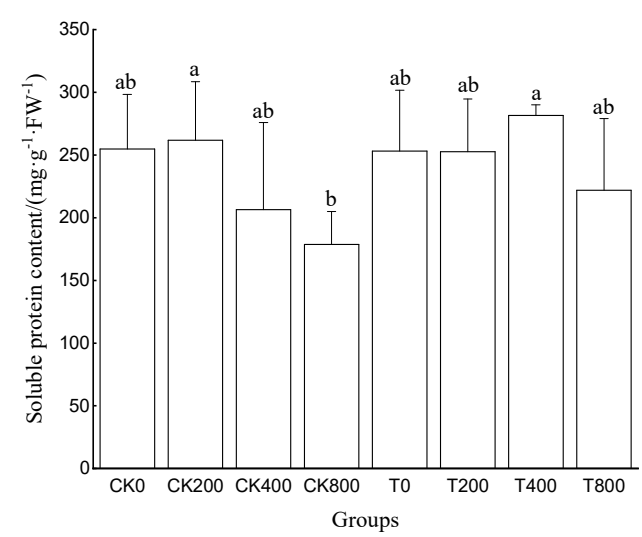

Figure 2. Effects of melatonin on the soluble protein of Abutilon theophrasti Medicus under $\mathrm{Cd}^{2+}$ stress 
Protein, as a biological macromolecule in cells, was one of the most important basic materials for the structure and function of organisms. As shown in Figure 2, after $5 \mathrm{~d} \mathrm{Cd}$ stress without melatonin, the SP content first increased and then decreased, and reached its maximum at CK200. After $5 \mathrm{~d} \mathrm{Cd}$ stress withmelatonin, the trend was the same as above, and reached its maximum at T400. It showed that melatonin pre-treatment increased SP content, and worked best on $400 \mu \mathrm{M}$ Cd stress, by $36.41 \%$.

Soluble protein was also an important osmotic adjustment substance in plants. Its content could show the strength of plant metabolism. Plants under adversity actively accumulate to improve plant osmotic balance. The study found that the SP content of abutilon seedlings increased first and then decreased under Cd stress, which might be due to an emergency response under adversity conditions. Melatonin treatment had no significant effect on the increase of soluble protein content.

\subsection{Effect of melatonin on content of MDA under cadmium stress}

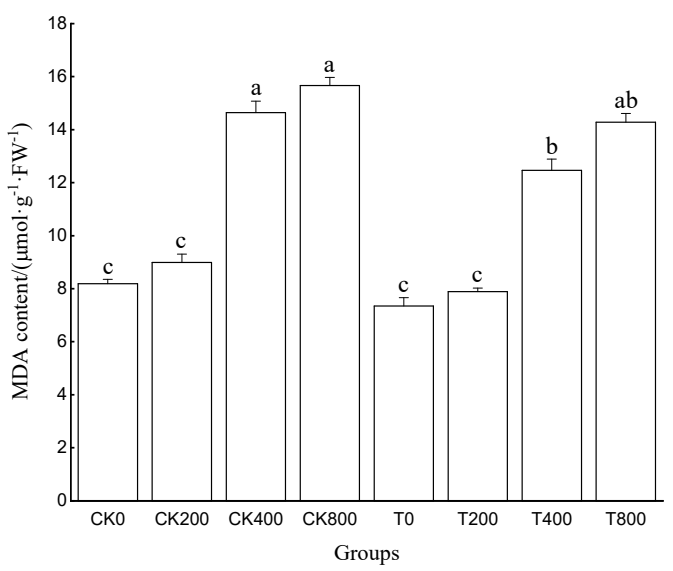

Figure 3. Effects of melatonin on the MDA content of Abutilon theophrasti Medicus under $\mathrm{Cd}^{2+}$ stress

MDA was a hallmark of cell membrane destruction. As shown in Figure 3, in the plant without melatonin pretreatments, $400 \mu \mathrm{M}$ and $800 \mu \mathrm{M}$ Cd stress respectively increased MDA as much as 1.79-folds and 1.91-folds compared to the control. Compared to the $400 \mu \mathrm{M} \mathrm{Cd}$ group, $0.1 \mathrm{mMmelatonin}$ pre-treatments resulted in MDA from $14.64 \mathrm{mmol} \cdot \mathrm{g}^{-1} \mathrm{FW}^{-1}$ to $12.47 \mathrm{mmol} \cdot \mathrm{g}^{-1} \mathrm{FW}^{-1}$ in the plants, significantly decreased $14.82 \%$.

Once subjected to stress, the balance of reactive oxygenmetabolism in plants will be disrupted, leading to an increase inROS content that causes membrane peroxidation and damageto the biofilm system. But melatonin was a substance with strong antioxidant capacity. It reduced oxidative damage by directly cleared $\cdot \mathrm{OH}$ and $\mathrm{H}_{2} \mathrm{O}_{2}$ molecules in cells and kept ROS at a relatively low level ${ }^{[15]}$. In this study, $0.1 \mathrm{mM}$ exogenous melatonin were applied to the MDA content under the different stresses of $\mathrm{Cd}$, indicating that $\mathrm{Cd}$ stress destroys the biofilm system, $0.1 \mathrm{mM}$ exogenous melatonin on plants reduced MDA content under Cd stress, thereby reducing cell damage and enhancing the ability of plants to resist abiotic stress. Melatonin had a certain stabilizing effect on alleviating the increase of MDA content caused by oxidative damage.

\subsection{Effect of melatonin on activity of POD under cadmium stress}

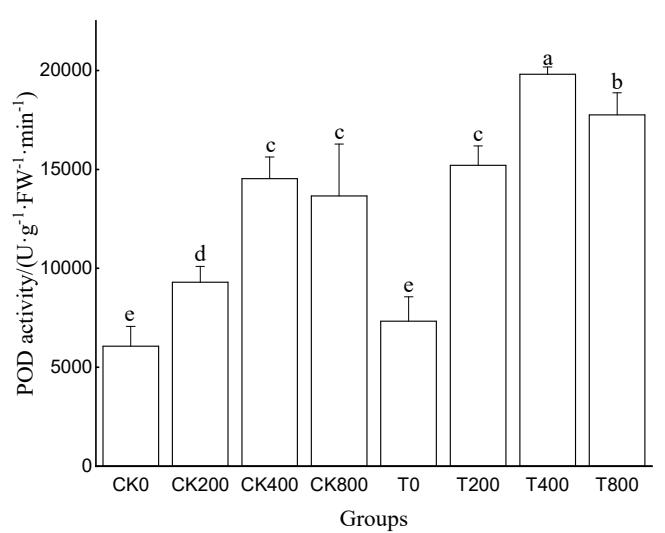

Figure 4. Effects of melatonin on POD content of Abutilon theophrasti Medicus under $\mathrm{Cd}^{2+}$ stress

Melatonin pre-treatment stimulated the activity of POD in leaves ofAbutilon seedlings under Cd stress. POD activity was the highest $\mathrm{T} 400$, and significantly higher than CK400 36.32\%. T200 and T800 respectively higher than same concentration control group $63.46 \%$ and $29.94 \%$. According to the results, $0.1 \mathrm{mM}$ melatonin had the best effect on $200 \mu \mathrm{M}$ Cd stress.

POD played an important role in scavenging reactive oxygen free radicals in plants. Due to its relatively high activity and sensitive response, POD had been regarded as anconsequence substance to maintain the balance of active oxygen system in the plants.In this experiment, POD activity increased significantly under Cdstress, indicating that the addition of $0.1 \mathrm{mMexogenous}$ melatonin showed similar changes in POD activity under different concentrations of $\mathrm{Cd}$ stress. It might be that POD corresponding gene transcription was not inhibited. Generated $\mathrm{H}_{2} \mathrm{O}_{2}$ further converted into $\mathrm{H}_{2} \mathrm{O}$ and $\mathrm{O}_{2}$, so that $\mathrm{H}_{2} \mathrm{O}_{2}$ did not damage to the plants, guaranteeing the regular growth. T200 had the most positive effect on pretreating melatonin. This was consistent with the conclusion of Zhang ${ }^{[16]}$ that exogenous melatonin could improve POD enzyme activity of rice seedlings under AS stress. The above results further indicated that $0.1 \mathrm{mM}$ exogenous melatonin had a certain inhibitory effect on membrane lipid peroxidation of abutilon seedlings under $\mathrm{Cd}$ stress, further maintaining the stability of active oxygen system of the plant to a certain extent.

\subsection{Effect of melatonin on accumulation under cadmium stress}

Table1. Variation of cadmium accumulation in each part

\begin{tabular}{|c|c|c|}
\hline Groups & $\begin{array}{c}\text { Cadmium content in } \\
\text { the ground } \\
(\mathrm{mg} / \mathrm{kg})\end{array}$ & $\begin{array}{c}\text { Cadmium content in } \\
\text { roots } \\
(\mathrm{mg} / \mathrm{kg})\end{array}$ \\
\hline
\end{tabular}




\begin{tabular}{|c|c|c|}
\hline CK200 & $6.83 \pm 0.08 \mathrm{Bf}$ & $87.27 \pm 0.58 \mathrm{Ae}$ \\
\hline CK400 & $40.71 \pm 0.60 \mathrm{Ba}$ & $189.65 \pm 0.71 \mathrm{Ac}$ \\
\hline CK800 & $40.38 \pm 1.51 \mathrm{Bb}$ & $315.27 \pm 0.64 \mathrm{Aa}$ \\
\hline $\mathrm{T} 200$ & $15.33 \pm 0.36 \mathrm{Be}$ & $58.28 \pm 5.31 \mathrm{Af}$ \\
\hline $\mathrm{T} 400$ & $22.64 \pm 0.44 \mathrm{Bd}$ & $108.73 \pm 1.10 \mathrm{Ad}$ \\
\hline $\mathrm{T} 800$ & $28.31 \pm 0.33 \mathrm{Bc}$ & $204.78 \pm 1.16 \mathrm{Ab}$ \\
\hline
\end{tabular}

Note: Different lowercase letters indicate significant differences among treatment groups in the same column. Different majuscule letters indicate significant differences among the ground and roots, the same as below $(\mathrm{P}<0.05)$.

Inaccordance with Table 1, Cd significantly increased $(\mathrm{P}<0.05)$ the Cadmium content in the ground and in the roots. The Cadmium content in the roots significantly higher than in the ground. This suggested that $\mathrm{Cd}$ mainly accumulated in the roots, only few shifted upward. It might be due to the roots directly contact with soil and a large number of microorganisms to heavy metal ions had good absorption and fixation, thus could enhance root of $\mathrm{Cd}$ enrichment capability. $\mathrm{Gu}^{[17]}$ found that melatonin reduced the accumulation of $\mathrm{Cd}^{2+}$ by up-regulating the transcription of PDR8 and HMA4 and down-regulating the transcription of Nramp6.It was speculated that melatonin might have a similar mechanism in the response to $\mathrm{Cd}$ stress, which needed to be further proved.Under the stress of $400 \mu \mathrm{M} \mathrm{Cd}$, the plants with $0.1 \mathrm{mM}$ melatonin pretreatment showed a highest decline $(\mathrm{P}<0.05)$ in the Cd concentration as much as $44.39 \%$ and $42.67 \%$ in the ground and roots in comparison with the control (the same Cd concentration without melatonin), respectively.

\subsection{Effect of melatonin on enrichment and transfer coefficients under cadmium stress}

Table2. Changes of cadmium enrichment and transfer coefficients

\begin{tabular}{|c|c|c|}
\hline Groups & Enrichment Factor & Transfer Factor \\
\hline CK200 & $6.83 \pm 0.05 \mathrm{~b}$ & $0.08 \pm 0.00 \mathrm{~d}$ \\
\hline CK400 & $8.36 \pm 0.05 \mathrm{a}$ & $0.21 \pm 0.00 \mathrm{~b}$ \\
\hline CK800 & $6.45 \pm 0.04 \mathrm{c}$ & $0.13 \pm 0.00 \mathrm{c}$ \\
\hline T200 & $5.34 \pm 0.37 \mathrm{~d}$ & $0.26 \pm 0.03 \mathrm{a}$ \\
\hline T400 & $4.77 \pm 0.02 \mathrm{e}$ & $0.21 \pm 0.01 \mathrm{~b}$ \\
\hline T800 & $4.23 \pm 0.03 \mathrm{f}$ & $0.14 \pm 0.00 \mathrm{c}$ \\
\hline
\end{tabular}

In accordance with Table $1, \mathrm{Cd}$ significantly decreased $(\mathrm{P}<0.05)$ the enrichment factor in the ground and in the roots. $200 \mu \mathrm{M} \mathrm{Cd}$ significantly increased the transfer factor. With pre-treatment of $0.1 \mathrm{mM}$ melatonin, the minimum BCF and TF were determined in $800 \mu \mathrm{M} \mathrm{Cd}$ stress. But without melatonin, the minimum TF was determined in $200 \mu \mathrm{M}$. T400 showed the most obvious BCF decline, was $42.94 \%$. Planting abutilon on medium contaminated soil effectively helped make more use of $\mathrm{Cd}$ contaminated soil and improve economic benefits. It seems that under $\mathrm{Cd}$ stress, melatonin could reduce the accumulation of cadmium in the plant, and increase the content of SP and the activity of antioxidant enzymes to resist the damage of $\mathrm{Cd}$.

\section{Conclusions}

(1) Pretreatment with $0.1 \mathrm{mM}$ melatonin significantly increased plant root activity, POD activity, chlorophyll and soluble protein content, andreduced MDA content. Therefore, melatonin could improve the tolerance of abutilon to cadmium.

(2) The $0.1 \mathrm{mM}$ melatonin pretreatment reduced the accumulation capacity of abutilon. Although it increased TF slightly, TF was significantly lower than BCF. Only a small amount of cadmium was transferred to the ground of the plant, and most of it accumulated in the root. It showed that melatonin had a certain effect on the healthy and economic production of abutilon.

\section{Acknowledgments}

The research was funded by the National Natural Science Foundation of China (31860176), the Key Research and Development Program of Shanxi Province (No. 20YF3FA037), the Key Research and Development Program of Shanxi Province (No. 2020ZDLSF06-06) and XAWLKYTD012. We were grateful to all anonymous reviewers whose comments improved the quality of the manuscript.

\section{References}

1. M. Benavides, S. Gallego, M.Brazilian Journal of Plant Physiology, 17 (2005)

2. A. Khan, S. Khan, M. Khan. Environmental Science and Pollution Research, 22(2015)

3. C. Hermans,J. Chen,F. Coppens.New Phytologist, 192 (2011)

4. S. Gallego, L. Pena, R. Barcia. Environmental Experimental Botany, 83 (2012)

5. M. Rizwan, S. Ali, T. Abbas. Ecotoxicology Environmental Safety, 130(2016)

6. T. Abbas, M. Rizwan,S. Ali. Environmental Science Pollution Research,25 (2017)

7. Z. Xu, Q. Li, P.Yang. Environmental Pollution,224 (2017).

8. C. Pape, K. Lüning. Trends Plant Science, 41(2006)

9. H. Kaur, S. Bhatla. Nitric Oxide, 59(2016)

10. G.Cui, X.Zhao,S. Liu.Plant Physiology and Biochemistry, 118(2017).

11. M.Nawaz, Y.Jiao, C.Chen. Journal of Plant Physiology, 220 (2018)

12. F.Hou. Experimental Course in Plant Physiology, (2015)

13. J. Chen, X. Wang.Experimental Guide to Plant Physiology, (2006) 
14. T.Wei, X. mLv, H.Jia. Journal of Environmental Management, 214(2018)

15. U.Fasih, L.Cai, A.Jeffrey.Ecotoxicology and Environmental Safety, 211 (2021)

16. S. Zhang, Y. Huang, S. Bao. Chinese Journal of Ecology, 39(2020)

17. Q.Gu, Z. Chen, X. Yu. Plant Science, 261 (2017) 